Case Report:

\title{
Retained Lumbar Intradiscal Surgical Knife Blade Retrieved Via Extended Extraforaminal Approach: Case Report and Review
}

Abolfazl Rahimizadeh ${ }^{1^{*}}$, Zahed Malekmohammdai $^{1} \mathrm{Q}$, Mahan Amirzadeh $^{1} \mathrm{Q}$, Shaghayegh Rahimizadeh ${ }^{1} \mathrm{Q}$, Walter Williamson $^{1}$

1. Pars Advanced and Minimally Invasive Medical Manners Research Center, Pars Hospital, Iran University of Medical Sciences, Tehran, Iran

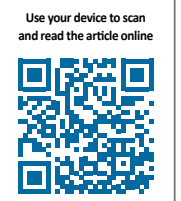

Citation Rahimizadeh A, Malekmohammdai Z, Amirzadeh M, Rahimizadeh S, Williamson W. Retained Lumbar Intradiscal Surgical Knife Blade Retrieved Via Extended Extraforaminal Approach: Case Report and Review. Iran J Neurosurg. 2021; 7(3):147-152. http://dx.doi.org/10.32598/irjns.7.3.5

: http://dx.doi.org/10.32598/irjns.7.3.5

(C) 08

Article info:

Received: 01 Jun 2021

Accepted: 29 Jun 2021

Available Online: $01 \mathrm{Jul} 2021$

Keywords:

Broken surgical blade, Complication, Extraforaminal, Foreign objects, Lumbar, Dscectomy, Transforaminal

\section{A B STRACT}

Background and Importance: During a lumbar discectomy, the surgical knife might be broken and embedded deeply within the disc space. In some cases, it may be impossible to remove the broken blade during the initial surgery despite allocating several hours for this purpose. This fact may justify a subsequent surgical session. However, the eventual retrieval of the broken scalpel during a second surgical encounter can likewise be a very daunting challenge.

Case Presantation: An L4-L5 discectomy in a young boy was complicated by the presence of an intradiscal broken surgical knife blade. The broken blade was successfully retrieved in a subsequent surgical session via the extended extraforaminal approach.

Conclusion: The occurrence of an intradiscal retained broken scalpel has been rarely discussed within medical literature. There exist a wide variety of different approaches used for such a needed retrieval. The extended extraforaminal corridor has yet to be described within the context of medical journalism.

\section{* Corresponding Author:}

Abolfazl Rahimizadeh, MD.

Address: Pars Advanced and Minimally Invasive Medical Manners Research Center, Pars Hospital, Iran University of Medical Sciences, Tehran, Iran Tel: +98 (21) 88952035

E-mail: a_rahimizadeh@hotmail.com 


\section{Highlights}

- Intradiscal broken surgical scalpel during discectomy is not a very rare scenario and most of us have experienced this complication, at least once.

- If this complication occurs, the surgeon should not become agitated, since he can easily use extraforaminal or extended extraforaminal route for retrieval of the hidden scalpel.

- Surgical microscope is necessary for retrieval.

- If the hidden object cannot be seen via these routes, the surgeon can change the corridor to transforaminal route with removal of the pars interarticularis. Obviously, pedicle screw fixation will be necessary either in the same or another session.

\section{Plain Language Summary}

The surgical knife might be broken during simple lumbar discectomy. The authors describe different approaches, including a new corridor for retrieval of the broken scalpel.

\section{Background and Importance}

or the retrieval of lumbar intradiscal

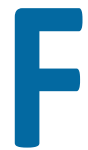
retained surgical instruments including the knife blade being presently addressed; the retroperitoneal corridor has remained the most preferred route for about the last two decades. More recently the extraforaminal and transforaminal routes were introduced [1-4]. Within the present time there are still a few more corridors and techniques being described $[5,6]$.

Herein, a young man with an intradiscal retained broken surgical scalpel is presented in whom the foreign body was removed via the extended extraforaminal route. The approach described in the current case provides a practical solution through which the broken blade might be discovered and retrieved in the future with much greater ease.

\section{Case Presentation}

A 23-year-old man was referred to our hospital after unsuccessful attempts for the retrieval of an intradiscal retained broken surgical knife blade. The incident that resulted in its being lodged had occurred during an L4L5 discectomy two days earlier.

The location of the broken blade could be suspected with the only available AP (anteroposterior) lumbar plane radiograph (Figure 1). However, the exact location of the shattered instrument could be clearly demonstrated within 3-D reconstructed computerized tomography scans (Figure 2).

\section{Surgical intervention}

The original incision was reopened, then by drilling the upper part of the cranial facet of $L 5$ and by the retraction of the $L 4$ root; the roof and uppermost part of lateral wall of the L4-L5 annulus were excised. This provided a large scene through which sufficient exposure to the remaining contents of the disc space and the broken knife blade located at the anterolateral part of the disc space could easily be determined. Subsequently, the broken blade was grasped and extracted (Figure 3). The postoperative course was uneventful and he was discharged a day later.

\section{Discussion}

The literature concerning the breakage of a surgical knife blade during a lumbar discectomy is scarce. Since its first description in 1994, a total number of 14 cases including the current case have been published (Table 1). It is important to note that there should have been plenty of cases with this iatrogenic complication which were never reported with respect to medicolegal implications.

A retained broken blade may remain stable and clinically silent. However, with ambulation, it usually has a tendency to migrate anteriorly toward the retroperitoneal cavity. This will inevitably lead to serious compli- 
Table 1. The breakage of the surgical knife blade during lumbar discectomy since 1994

\begin{tabular}{|c|c|c|c|c|c|}
\hline Authors & Year & Sex & Age & Level & Surgical Approach \\
\hline Amirjamshidi et al. [1] & 1994 & & & & Anterior retroperitoneal \\
\hline Amirjamshidi et al. [1] & 1994 & & & & Anterior retroperitoneal \\
\hline Amirjamshidi et al. [1] & 1994 & & & & Anterior retroperitoneal \\
\hline Amirjamshidi et al. [1] & 1994 & & & & Anterior retroperitoneal \\
\hline Rahimizadeh et al. [2] & 2013 & & & & Transforaminal, with microscope \\
\hline De Praetere et al. [9] & 2014 & M & 44 & L5-S1 & Migrated to heart, open heart surgery \\
\hline Rahimizadeh \& Hadadi [3] & 2016 & $\mathrm{~F}$ & & L5-S1 & Transforaminal with pars removal, with microscope \\
\hline Limbachia \& Gandhi [11] & 2017 & M & 40 & L5-S1 & Retroperitoneal, laparascopic \\
\hline Koutserimpas et al. [12] & 2019 & $\mathrm{~F}$ & 54 & L4-L5 & Robotic retropeitoneal approach \\
\hline Zheng \& Wang [6] & 2020 & $\mathrm{~F}$ & 56 & L4-L5 & Posterior, conventional, with arthroscope \\
\hline Wu et al. [7] & 2020 & M & 49 & L2-L3 & Posterior, conventional with microscope \\
\hline Agrawal et al. [5] & 2020 & M & 29 & L1-L2 & Posterior, conventional with microscope \\
\hline Barbero-Aznarez et al. [10] & 2021 & M & 54 & L4-L5 & Lateral, transpsoas \\
\hline Current case & 2021 & M & & L4-L5 & Extended extraforaminal, with microscope \\
\hline
\end{tabular}

cations $[1,7]$. Migration of the broken blade into the pelvic cavity and penetration of the vena cava or its still further movement toward the left atrium are rather apt

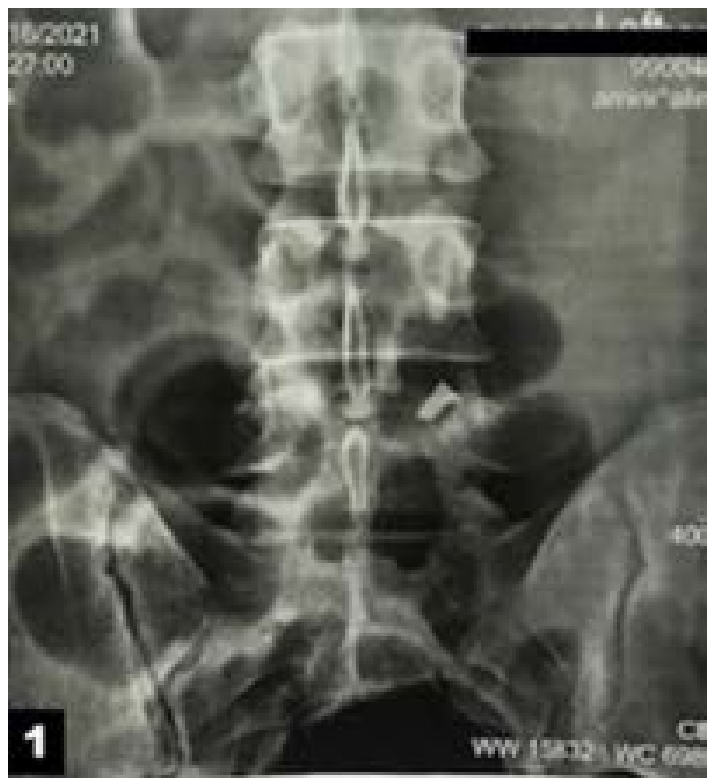

Figure 1. AP lumbar spine radiograph

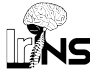

The only available radiograph shows that the broken blade is on the left side. examples of the deadly hazards of a possible migration scenario $[8,9]$.

Removal of a retained broken surgical knife blade is an admittedly challenging issue. Despite a variety of approaches and different corridors which have been proposed; there are no unique guidelines to properly address the management of this complication. For years the retroperitoneal approach was accepted as the corridor of choice for this purpose. Then the more suitable extraforaminal approach was described in 2013 [1, 2]. Later, the transforaminal approach with pars removal was introduced for its relative ease of extraction. Recently, Zheng and Wang were able to remove a broken blade with arthroscopic assistance [6]. The extended extraforaminal approach which was utilized in the current case provides a large visual field which might obviate the necessity of a pars removal as well as the offending instrumentation (Figure 4). In 2021, Barbero-Aznarez et al. successfully employed the paravertebral transposition approach for this purpose [10]. We believe that in the majority of cases, with consideration given for the familiarity of the neurosurgeons; the broken blade can nearly always be retrieved via one of these posterior corridors. Apparently, a posterior approach via an initial 


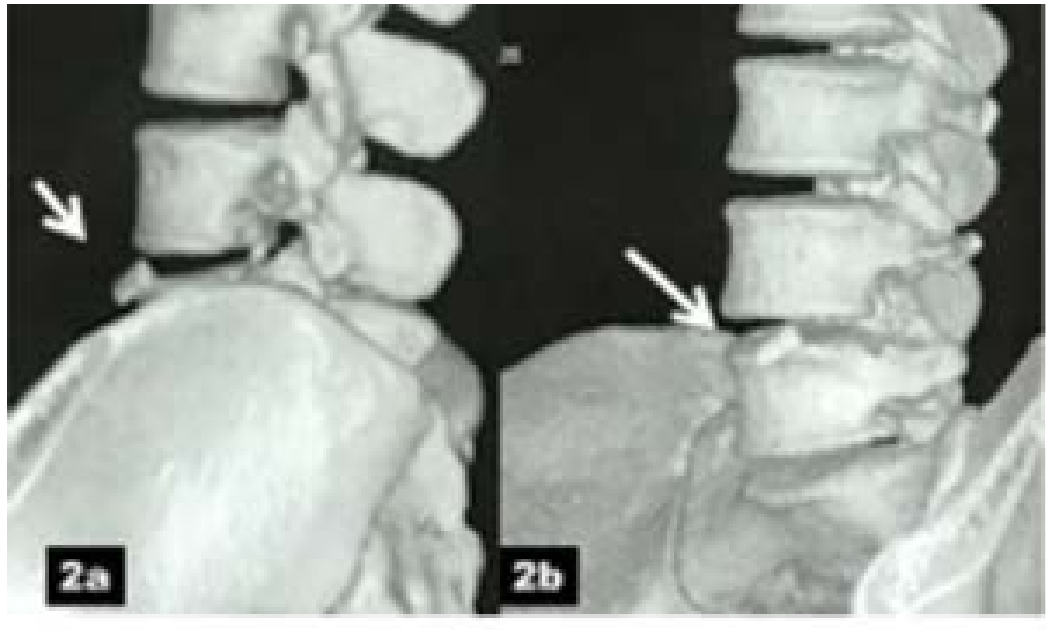

$2 \mathrm{a}$

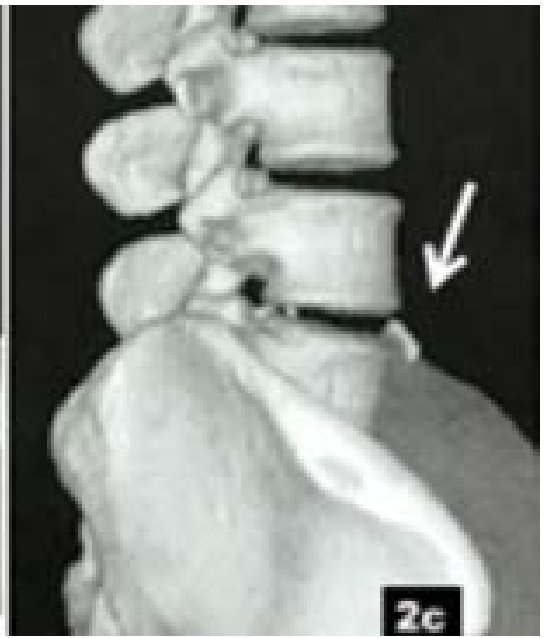

INS

Figure 2. 3D lumbosacral CT scan

Images show the exact location of the blade; two-thirds of the blade have passed anterior longitudinal ligament (White arrows) (a, b, and c).

incision is psychologically better accepted by the patients and his/her relatives. However, if most of the broken blade traverses through the anterior longitudinal ligament or is lodged entirely out of the disc space, then its retrieval via a conventional retroperitoneal approach will be required [1, 7]. Recently, laparoscopic approach and robot-assisted laparoscopy have been described as alternative options $[11,12]$.

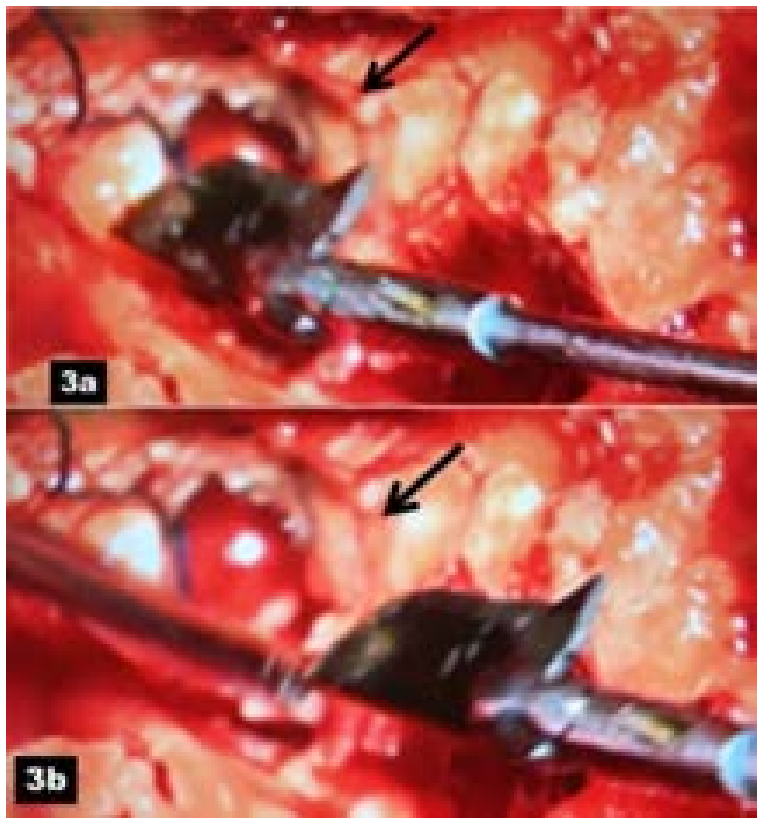

INS

Figure 3. Intraoperative photograph

A \& B: Showing removal of the blade via extended extraforaminal corridor. L4 root located is cranial to the surgical entry (Black arrows).

\section{Conclusion}

With consideration of the potential hazards of an intradiscal broken scalpel, its removal either in the initial or within a subsequent surgical session is medically mandatory. The extended extraforaminal route might be safest as well as the easiest modified corridor for all intradiscal retained foreign bodies including a broken blade.

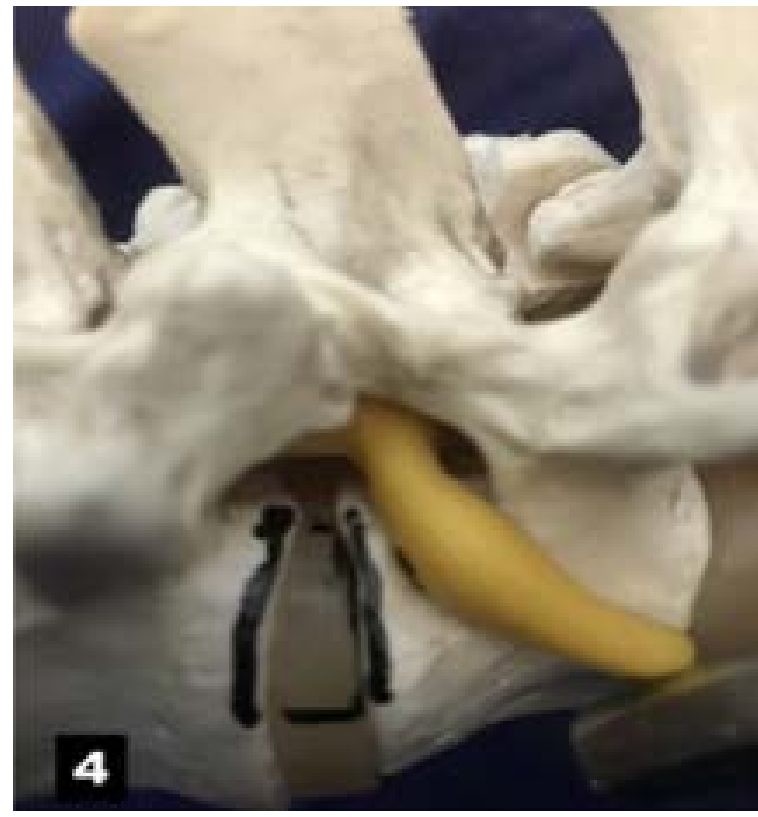

Figure 4. Schematic photograph showing extended extraforaminal opening L4 root is retracted cranially. 


\section{Ethical Considerations}

Compliance with ethical guidelines

Written consent was obtained from the patient to publish the history and her corresponding radiological images.

Funding

This research did not receive any grant from funding agencies in the public, commercial, or non-profit sectors.

Authors' contributions

Conception and design: Abolfazl Rahimizadeh, Zahed Malekmohammdai; Data collection: Abolfazl Rahimizadeh, Zahed Malekmohammdai, Mahan Amirzadeh; Data analysis and interpretation: Abolfazl Rahimizadeh, Shaghayegh Rahimizadeh; Drafting the article: Abolfazl Rahimizadeh, Mahan Amirzadeh; Critically revising the article: Shaghayegh Rahimizadeh, Walter Williamson; Reviewing submitted version of manuscript: Abolfazl Rahimizadeh, Zahed Malekmohammdai; Approving the final version of the manuscript: Abolfazl Rahimizadeh, Walter Williamson.

\section{Conflict of interest}

The authors declared no conflict of interest.

\section{Acknowledgements}

The authors would like to offer their special thanks to Dr. Naser Asgari for his useful comments.
[5] Agrawal Y, Sharma S, Chopra S, Purohit DK. Retrieval of a retained broken scalpel blade from lumbar intervertebral disc space: A case report. Romanian Neurosurgery. 2017; 31(1):129-32. [DOI:10.1515/romneu-2017-0020]

[6] Zheng G bin, Wang Z. Removal of the deeply located intradiskal broken knife blade with arthroscopic assistance: Case report and literature Review. World Neurosurgery. 2020 137:272-5. [DOI:10.1016/j.wneu.2020.01.221] [PMID]

[7] Wu C, Wu MH, Lee CY, Li YY, Huang, TJ. Retrieval of a broken surgical scalpel blade with a dangerous anterior migration in treating a herniated disk along with an osteophyte: A case report. Formosan Journal of Musculoskeletal Disorders. 2020; 11(3):123-7. [DOI:10.6492/FJMD.202008_11(3).0005]

[8] Li H, Wang W, He A. [A broken sharp scalpel left in an intervertebral space and slipped to pelvic cavity during an operation of intervertebral disc displacement (Chinese)]. Hunan yi ke da xue xue bao. 1998; 23(6):614. [PMID]

[9] De Praetere H, Vanden Eycken C, Meuris B, Herijgers P. Migration of a broken scalpel into the heart after spine surgery. Interactive Cardiovascular and Thoracic Surgery. 2014; 18(4):527-9. [DOI:10.1093/icvts/ivt553] [PMID] [PMCID]

[10] Barbero-Aznarez P, Bucheli-Peñafiel C, Olmos-Francisco E, Lorente-Muñoz A, Cortés-Franco S. Broken surgical blade retrieval following lumbar discectomy through paravertebral/ lateral transpsoas approach: A case report. Surgical Neurology International. 2021; 12:25. [DOI:10.25259/SNI_880_2020] [PMID] [PMCID]

[11] Limbachia D, Gandhi P. Laparoscopic retrieval of a foreign body (broken surgical knife) from retroperitoneal space: An interesting case. Gynecology and Minimally Invasive Therapy. 2017; 6(4):193-4. [DOI:10.1016/j.gmit.2016.10.003] [PMID] [PMCID]

[12] Koutserimpas C, Ioannidis A, Konstantinidis M, Athanasopoulos P, Antonakopoulos F, Konstantinidis K. Robot-assisted removal of a broken scalpel blade following discectomy. Case Reports in Surgery. 2019; 2019:8609246. [DOI:10.1155/2019/8609246] [PMID] [PMCID]

\section{References}

[1] Amirjamshidi A, Mehrazin M, Abbassioun K, Ketabtchi E. Retained broken knife blades within the disc space. Spine. 1994; 19(8):981-4. [DOI:10.1097/00007632-199404150-00018] [PMID]

[2] Rahimizadeh A, Ghorbani E, Rahimizadeh S. Transforaminal retrieval of intradiscal retained broken surgical knife blade. Spine. 2013; 38(20):E1278-81. [DOI:10.1097/BRS.0b013e31829ef4d7] [PMID]

[3] Rahimizadeh A, Haddadi K. Is transforaminal retrieval of intradiscal deeply seated broken surgical knife blade all time pars sparing? A case report. International Journal of Surgery Case Reports. 2016; 19:131-3. [DOI:10.1016/j.ijscr.2015.12.040] [PMID] [PMCID]

[4] Rahimizadeh A, Rahimizadeh S, Amirzadeh M. Intradiscal retained broken blade of A disc rongeur. Journal of Spine \& Neurosurgery. 2017; 6(3):1-3. [DOI:10.4172/2325-9701.1000273] 
This Page Intentionally Left Blank 